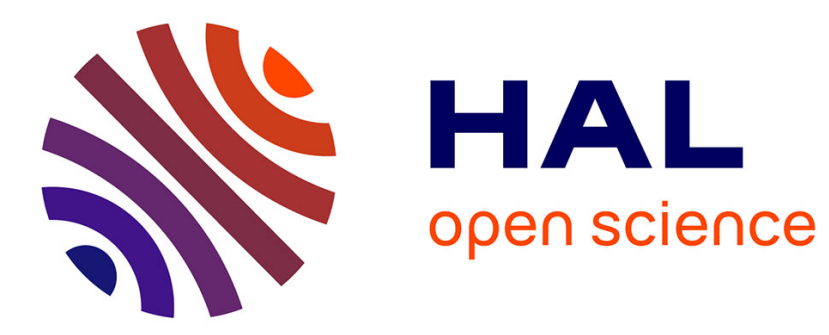

\title{
Integrated method for tool path generation in five-axis sculptured surface machining
}

\author{
Jianhua Fan, A Ball
}

\section{To cite this version:}

Jianhua Fan, A Ball. Integrated method for tool path generation in five-axis sculptured surface machining. International Journal of Production Research, 2011, 10.1080/00207543.2010.528062 . hal00673192

\section{HAL Id: hal-00673192 \\ https://hal.science/hal-00673192}

Submitted on 23 Feb 2012

HAL is a multi-disciplinary open access archive for the deposit and dissemination of scientific research documents, whether they are published or not. The documents may come from teaching and research institutions in France or abroad, or from public or private research centers.
L'archive ouverte pluridisciplinaire HAL, est destinée au dépôt et à la diffusion de documents scientifiques de niveau recherche, publiés ou non, émanant des établissements d'enseignement et de recherche français ou étrangers, des laboratoires publics ou privés. 


\section{Integrated method for tool path generation in five-axis sculptured surface machining}

\begin{tabular}{|r|l|}
\hline Journal: & International Journal of Production Research \\
\hline Manuscript ID: & TPRS-2010-IJPR-0447.R1 \\
\hline Manuscript Type: & Original Manuscript \\
\hline $\begin{array}{r}\text { Date Submitted by the } \\
\text { Author: }\end{array}$ & 03-Sep-2010 \\
\hline Complete List of Authors: & $\begin{array}{l}\text { Fan, Jianhua; The University of Birmingham, Mechanical } \\
\text { Engineering } \\
\text { Ball, A; The University of Birmingham, Mechanical Engineering }\end{array}$ \\
\hline Keywords: & CNC MACHINING, CAM, MACHINING PROCESSES \\
\hline Keywords (user): & $\begin{array}{l}\text { Five-axis; Machining; Tool path generation; Cutter orientation; } \\
\text { Machined strip width }\end{array}$ \\
\hline
\end{tabular}


International Journal of Production Research

Vol. 00, No. 00, 00 Month 2009, 1-21

\title{
RESEARCH ARTICLE
}

\section{Integrated method for tool path generation in five-axis sculptured surface machining}

\author{
Jianhua Fan* and Alan Ball \\ Geometric Modelling Group, School of Mechanical Engineering, The University of \\ Birmingham, Edgbaston, Birmingham, B15 2TT, UK \\ (Received 00 Month 200x; final version received 00 Month 200x)
}

Five-axis machining allows continuous adjustment of cutter orientation along a tool pass. Unfortunately, the flexibility has not been fully exploited due to the separate consideration of tool path generation and cutter orientation in current machining methods. This paper presents an integrated method (IM) for tool path generation, which is tightly integrated with the orientation strategy, to minimise tool path length under the constraint of smooth cutter orientation. Distinctively, cutter orientation along a tool pass is optimised by balancing considerations of maximum material removal and smoothness of cutter movement. Further, the intervals between successive tool passes are maximised according to the optimised orientation. In the paper, the IM is combined with the quadric method, a recently developed cutter orientation strategy, for iso-parametric machining with a flat-end cutter. However, the method could be applied to other orientation strategies with different machining mechanisms and cutter types. Simulated examples illustrate that the IM is more efficient in machining than established methods.

Keywords: Five-axis; Machining; Tool path generation; Cutter orientation; Machined strip width

${ }^{*}$ Corresponding author. Email: jianhua.fan@gmail.com

ISSN: 1478-6451 print/ISSN 1478-646X online

(C) 2009 Taylor \& Francis

DOI: $10.1080 / 1478645 Y$ Yxxxxxxx

http://www.informaworld.com 


\section{Introduction}

Five-axis machining has been increasingly used in the manufacture of sculptured surfaces. Compared to 3-axis machining, it provides two extra degrees of freedom, which allow continuous adjustment of cutter orientation along tool passes. Consequently, a significant improvement in machining efficiency and surface finish can be achieved (Choi and Jerard 1998). Unfortunately, these benefits have not been fully realised due mainly to limited geometric research on surface manufacturing (Yoon et al. 2002). One key issue in tool path generation is how to optimise the two orientation angles along tool passes for maximising the machining efficiency.

Much research effort has been concentrated on optimising cutter orientation at one cutter contact (CC) point to locally maximise material removal (Fan and Ball 2008). However, the local solutions are not guaranteed to vary smoothly along each tool pass. Accordingly, there can be abrupt changes in cutter movement. Such abrupt changes may generate self-intersections and twists on the surfaces swept by the cutter, which lead to poor surface finish and low machining accuracy. Also, the abrupt changes induce rapid increases of cutting force, which might even damage the machined surface, cutter and machine tool. Thus, both material removal and cutter movement need to be considered in determining cutter orientation along the tool passes and, ideally, the cutter orientation and tool path generation should be integrated. Unfortunately, they are approached as two separate tasks in current machining methods.

As a contribution to improving machining efficiency, this paper develops an integrated method (IM) for tool path generation, which is tightly integrated with an orientation strategy. In the IM, cutter orientation is optimised by balancing considerations of maximum material removal and smoothness of cutter movement. Further, each successive tool pass is positioned as far as permissible from the preceding one. Then numerical simulations, comparing the IM with existing methods, show a significant reduction in the number of tool passes.

\subsection{Basic assumptions and concepts}

To avoid ambiguity in the discussion throughout the paper, some basic assumptions and concepts of cutter orientation and tool path generation are introduced in this subsection.

The scope of the research is limited to a geometric study of surface machining under the following assumptions:

- Design surface $\mathbf{S}(u, v)$ is a sculptured surface defined on a unit square $(0 \leq u, v \leq 1)$ with curvature continuity $\left(\mathrm{G}^{2}\right)$ (Farin 2002). This encompasses all (non-degenerate) cubic and higher order B-spline/NURBS surfaces, but any compound surface (Choi and Jerard 1998) with lower order continuity or irregular topology has to be machined patch-by-patch.

- Cutter is a flat-end cutter and its spindle speed is much higher than the feed rate. Thus, material can be considered to be removed by the sweeping motion of a cylindrical surface, the cutter surface $\mathbf{C}$.

- Cutter size has been selected appropriately (Jensen et al. 2002).

- Potential collisions on the side of $\mathbf{C}$ have been avoided by restricting the cutter orientation, but possible interference on the bottom of $\mathbf{C}$ needs to be checked.

- Step-forward distance is sufficiently small and tool passes $\mathbf{P}_{i}, i=1, \ldots, n$, along which $\mathbf{C}$ moves on $\mathbf{S}$, are parametric curves of $\mathbf{S}$. Thus, iso-parametric machining (Choi and Jerard 1998) is studied and, without loss of generality, $\mathbf{P}_{i}$ has constant $v$-value denoted by $v_{i}$. 


\section{eptember 3, 2010 \\ 23:36 International Journal of Production Research \\ International Journal of Production Research}

International Journal of Production Research

- Allowable deviation of the machined surface $\mathbf{M}$ from $\mathbf{S}$, referred to as machining tolerance $\varepsilon$, is positive and consistent with that in the finishing stage (Rao and Sarma 2000).

Cutter orientation is used to specify how $\mathbf{C}$ is placed relative to $\mathbf{S}$ at their contact point, the cutter contact $(C C)$ point. As depicted in figure 1, the orientation is described in a local machining coordinate system (LMCS, $\mathrm{X}_{\mathrm{M}}-\mathrm{Y}_{\mathrm{M}}-\mathrm{Z}_{\mathrm{M}}$ ), where the $\mathrm{Z}_{\mathrm{M}}$-axis is along the surface normal $\mathbf{N}$ and the $\mathrm{X}_{\mathrm{M}}$-axis is in the cutting direction. The cutter is first rotated a lead angle $\alpha$ around the $\mathrm{Y}_{\mathrm{M}}$-axis, and then a screw angle $\beta$ around the $\mathrm{Z}_{\mathrm{M}}$-axis. In general, $0 \leq \alpha \leq \pi / 2,-\pi / 2<\beta<\pi / 2$, but may be further restricted by collision avoidance.

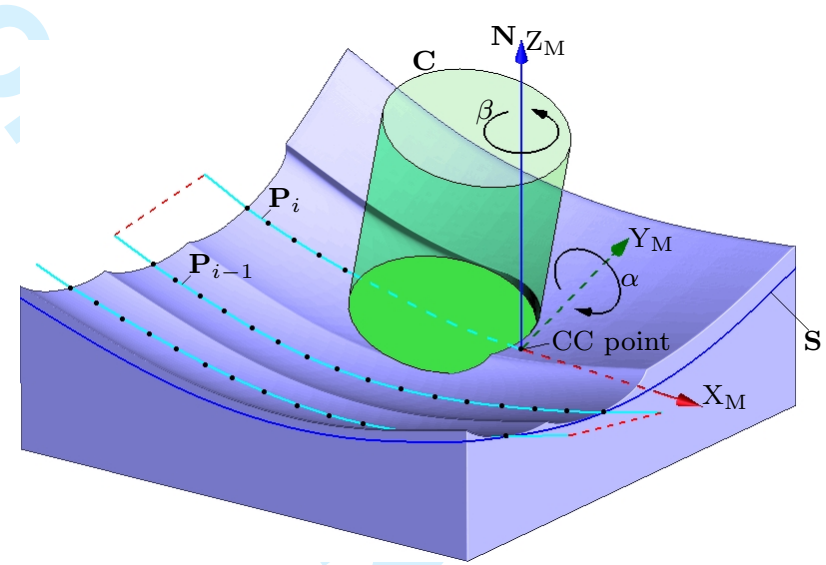

Figure 1. Cutter orientation in 5-axis machining

Cutter orientation can be assessed locally by the machined strip width $w$, which is defined as follows: Let an error surface $\mathbf{E}$ denote the offset of $\mathbf{S}$ by a distance $\varepsilon$. Then an area on $\mathbf{E}$ bounded by the intersection curves with $\mathbf{C}$ is a machined region $\mathbf{G}$. The maximum span of $\mathbf{G}$ along the $\mathrm{Y}_{\mathrm{M}}$-axis is the width $w$. For machining efficiency at the $\mathrm{CC}$ point, an optimal orientation is desired to maximise $w$ and give no gouging.

As $\mathbf{C}$ moves along $\mathbf{P}_{i}$, it sweeps out a cutter swept surface $\mathbf{W}_{i}$. A sequence of $\mathbf{W}_{i}$ removes material to generate the machined surface $\mathbf{M}$. To ensure the machining deviation, the distance from $\mathbf{M}$ to $\mathbf{S}$, is smaller than $\varepsilon, \mathbf{M}$ must lie between $\mathbf{S}$ and $\mathbf{E}$. Define an area on $\mathbf{E}$ bounded by the intersection curves with $\mathbf{W}_{i}$ as the machined strip $\mathbf{I}_{i}$. Then the strip $\mathbf{I}_{i}$ can also be considered as an envelope of the machined region $\mathbf{G}$. The strip $\mathbf{I}_{i}$, region $\mathbf{G}$ and width $w$ are intuitively defined in Euclidean space. However, in iso-parametric machining, it is convenient to define them in the parametric space of $\mathbf{S}$. Thus, the machining can be considered as a process of overlapping a sequence of machined strips in the parametric space as illustrated in figure 2 .

To improve the machining efficiency in iso-parametric machining, the length of tool path or, equivalently, the number of tool passes needs to be reduced. Accordingly, research is directed to maximising the widths of individual strips $\mathbf{I}_{i}$ and the step-over distances between successive strips $\mathbf{I}_{i-1}$ and $\mathbf{I}_{i}$, whilst ensuring there are only smooth changes in the cutter orientation and no gaps between the strips. 


\subsection{Previous work}

There has been much research effort to optimise the cutter orientation at a CC point in 5-axis sculptured surface machining. The published algorithms can be classified broadly into local and global methods (Fan and Ball 2008). In the local methods (Vickers and Quan 1989, Bedi et al. 1997, Rao and Sarma 2000, Jensen et al. 2002, Yoon et al. 2002), only normal curvatures of $\mathbf{C}$ ( or $\mathbf{W}_{i}$ ) and $\mathbf{S}$ are considered to orient $\mathbf{C}$. The main disadvantage of the local methods is that there could still be rear gouging, and consequently a secondary iterative gouge-check and correction algorithm has to be implemented (Gray et al. 2005). The global methods overcome the disadvantage by using an area of $\mathbf{S}$ beneath $\mathbf{C}$ to determine the orientation (Warkentin et al. 2000, Gray et al. 2003, 2005, Hosseinkhani et al. 2007, Fan and Ball 2008). In particular, the quadric method (QM) (Fan and Ball 2008) exploits fully the orientation angles $(\alpha, \beta)$ with respect to the machined strip width $w$. Further, the width evaluation is involved in the method, and its approximation error is conservative and acceptably small. Thus, it is adopted to orient the cutter along tool passes in this study.

Cutter orientation only aims to maximise the material removal at one CC point in a specified cutting direction. The distribution and ordering of $\mathrm{CC}$ points over the entire design surface is studied in tool path generation. In general, a tool path can be topologically classified as a raster or a spiral pattern (Choi and Jerard 1998). The spiral tool path keeps the cutter continuously removing material but is more restricted in its applicability. For machining a general sculptured surface, the raster pattern is always adopted and the tool path can be further categorised as an iso-parametric, iso-planar or iso-scallop method of construction (Chiou and Lee 2002). The iso-scallop method has a shorter tool path than the others (Chiou and Lee 2002, Li and Feng 2004, Tournier and Duc 2005), but may have more broken tool passes and fail to converge in the numerical computation (Rao et al. 2000, Tournier and Duc 2005). Therefore, the iso-parametric and iso-planar methods are most widely and almost exclusively used in industry.

Tool path generation methods and cutter orientation strategies are currently approached as two separate tasks. In order to maximise the step-over distance, the cutter orientation at each $\mathrm{CC}$ point is optimised locally without considering its 


\section{eptember 3,2010

variation between neighbouring points (Choi and Jerard 1998, Jensen et al. 2002, Li and Feng 2004). Accordingly, there could be some abrupt changes in orientation along a tool pass. Jun et al. (2003) developed a method to avoid the abrupt changes by searching alternative orientations in the machining configuration space and minimising the variation in cutter orientation. Wang and Tang (2007) further improved the method by limiting the rate of change of orientation angles in a given range. However, the second derivatives of the orientation angles have not been controlled and the impact on the step-over distance when limiting the angles has not been considered in either method.

The idea of integrated tool path generation was first presented by Rao et al. (2000). In their method, cutter orientation is selected based on its contribution to the complete tool pass. However, the width evaluation from the intersections of normal vectors with the cutter gives no consideration to the error introduced by the surface discretisation, nor the computational expense if dense vectors are adopted (Choi and Jerard 1998). Further, the variation of the screw angle between two adjacent $\mathrm{CC}$ points is minimised but not smoothed along the tool pass.

Based on the previous work, this paper proposes an integrated method (IM) for tool path generation, which aims to minimise the number of tool passes in surface machining under the constraint of smooth cutter orientation. The rest of the paper is organised as follows: The machined strip is represented in parametric space in Section 2. Then, the screw angle along a tool pass is expressed in B-spline form and optimised by balancing considerations of the width and the smoothness of cutter movement in Section 3. Further, the next tool pass is positioned as far as permissible from the previous one in Section 4. Finally, the proposed method is illustrated with numerical examples in Section 5, followed by the research conclusions in Section 6 .

\section{Machined strip along a tool pass}

To optimise the cutter orientation along a tool pass and control the overlap between two adjacent machined strips in iso-parametric machining, it is convenient to represent the strips and their widths in the parametric space of the design surface $\mathbf{S}$.

\subsection{Parametric representation of machined strip}

As shown in figure 3 , let $\mathbf{D}_{\mathbf{1}}$ and $\mathbf{D}_{2}$ denote the two width points, which determine the machined strip width at a CC point. They are the intersections between the error surface $\mathbf{E}$ and the bottom circle of the cutter $\mathbf{C}$, and can be evaluated by the quadric method (QM) in Euclidean space (Fan and Ball 2008). Suppose that the surface parameters, corresponding to $\mathbf{D}_{1}$ and $\mathbf{D}_{2}$, are respectively $\left(u_{1}, v_{1}\right)$ and $\left(u_{2}, v_{2}\right)$. Then, $\mathbf{D}_{1}$ lies on the straight line passing through $\mathbf{S}\left(u_{1}, v_{1}\right)$ in the normal direction $\mathbf{N}\left(u_{1}, v_{1}\right)$. Thus

$$
\mathbf{S}\left(u_{1}, v_{1}\right)+h_{1} \mathbf{N}\left(u_{1}, v_{1}\right)=\mathbf{D}_{1}
$$

where $h_{1}$ is the distance between $\mathbf{S}\left(u_{1}, v_{1}\right)$ and $\mathbf{D}_{1}$. Ideally, $h_{1}$ should be equal to the machining tolerance $\varepsilon$. However, in the QM, $\mathbf{D}_{1}$ is evaluated by replacing the error surface $\mathbf{E}$ with a translated quadric, which means $h_{1}$ is always smaller than $\varepsilon$. Consequently, equation (1) consists of three nonlinear component equations in three unknowns $u_{1}, v_{1}$, $h_{1}$, and has generally to be solved by a numerical method, such as the Newton-Raphson 


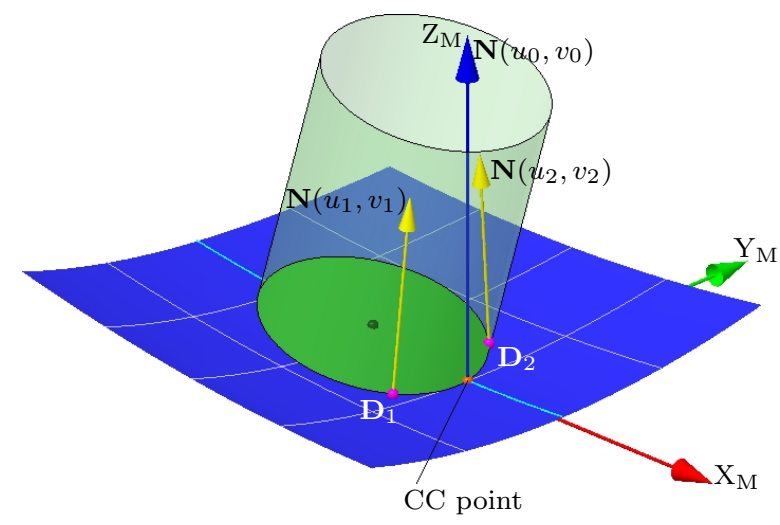

Figure 3. Parametric representation of width points

method. An initial estimate of $\mathbf{S}\left(u_{1,0}, v_{1,0}\right)$ is evaluated from that of the previous width point, and an initial estimate of $h_{1,0}$ is set as $\varepsilon$.

Let $u_{1, q}, v_{1, q}, h_{1, q}$ be the $q$ th approximation of a root of (1) and $\Delta u, \Delta v, \Delta h$ the respective errors. Then

$$
\mathbf{S}\left(u_{1, q}+\Delta u, v_{1, q}+\Delta v\right)+\left(h_{1, q}+\Delta h\right) \mathbf{N}\left(u_{1, q}+\Delta u, v_{1, q}+\Delta v\right)=\mathbf{D}_{1}
$$

Expanding (2) in a Taylor series, taking the first order approximation and rearranging, yields

$$
a_{1, q} \Delta u+b_{1, q} \Delta v+c_{1, q} \Delta h=d_{1, q},
$$

where

$$
\begin{aligned}
& a_{1, q}=\mathbf{S}_{u}\left(u_{1, q}, v_{1, q}\right)+h_{1, q} \mathbf{N}_{u}\left(u_{1, q}, v_{1, q}\right), \quad b_{1, q}=\mathbf{S}_{v}\left(u_{1, q}, v_{1, q}\right)+h_{1, q} \mathbf{N}_{v}\left(u_{1, q}, v_{1, q}\right), \\
& c_{1, q}=\mathbf{N}\left(u_{1, q}, v_{1, q}\right), \quad d_{1, q}=\mathbf{D}_{1}-\mathbf{S}\left(u_{1, q}, v_{1, q}\right)-h_{1, q} \mathbf{N}\left(u_{1, q}, v_{1, q}\right) .
\end{aligned}
$$

By solving the linear equation (3), $\Delta u, \Delta v$ and $\Delta h$ are obtained, and the next approximation is determined by

$$
u_{1, q+1}=u_{1, q}+\Delta u, \quad v_{1, q+1}=v_{1, q}+\Delta v, \quad h_{1, q+1}=h_{1, q}+\Delta h .
$$

The iterative procedure for finding the root of (1) is terminated if

$$
\left|d_{1, q}\right|<e_{1} \quad \text { or } \quad\left|\mathbf{S}\left(u_{1, q+1}, v_{1, q+1}\right)-\mathbf{S}\left(u_{1, q}, v_{1, q}\right)\right|<e_{2},
$$

where $e_{1}, e_{2}>0$ are the predefined accuracies of the point evaluation. Hence, the parameters $\left(u_{1}, v_{1}\right)$ of the width point $\mathbf{D}_{1}$ are obtained. Similarly, $\left(u_{2}, v_{2}\right)$ of $\mathbf{D}_{2}$ can be evaluated.

As the cutter $\mathbf{C}$ moves along the tool pass, the machined strip is bounded by two sets of width points. Accordingly, the strip can be expressed in the parametric space of the design surface $\mathbf{S}$.

\section{2. $\quad$ Parametric representation of width}

Suppose that the cutter $\mathbf{C}$ moves along a tool pass $\mathbf{P}_{i}$ with a constant $v$, and the parameters of the two width points are $\left(u_{1}, v_{1}\right)$ and $\left(u_{2}, v_{2}\right)$ at a CC point. Then 


\section{ptember 3, 2010 \\ International Journal of Production Research}

$w=\left|v_{1}-v_{2}\right|$ is referred to as the parametric representation of the machined strip width at this location. The formulation takes no account of any difference in the values of $u_{1}$ and $u_{2}$, but this can have little practical effect in parametric machining unless $v_{1}$ and $v_{2}$ are changing a lot between successive CC points on the tool pass. Further, it should be pointed out that the values of $u_{1}$ and $u_{2}$ are taken into account in Section 4.1, when ensuring successive machined strips overlap. The machining is therefore guaranteed to cover the whole surface although there could be a slight compromise of the machining efficiency.

In the quadric method (QM) for 5-axis sculptured surface machining (Fan and Ball 2008), the design surface $\mathbf{S}$ is approximated locally by two quadrics. An upper quadric lying above $\mathbf{S}$ is used to orient the cutter and a lower quadric below $\mathbf{S}$ is used to evaluate the machined strip width.

To maximise the width, for a given screw angle $\beta$, the lead angle $\alpha$ is optimised by placing the cutter $\mathbf{C}$ as close as possible to the upper quadric. Since the bottom plane of $\mathbf{C}$ intersects the upper quadric in a conic, the optimal $\alpha$ can be obtained based on the intersections of the bottom circle of $\mathbf{C}$ and the conic. there are in general four roots. Note that the circle and the conic share a common tangent direction at the CC point which accounts for two of the roots and the remaining two determine whether $\alpha$ should be increased to avoid gouging or decreased for cutting more material. The optimal solution for $\alpha$ is where the remaining two roots are equal and is referred to as two contact machining.

The machined strip width is determined by considering the distance of the surface below the bottom circle of the cutter $\mathbf{C}$. It is guaranteed in two contact machining that all the distances are positive and the critical points are where the distances are equal to the machining tolerance $\varepsilon$. There are at most four such points, which can be found by translating the lower quadric in the normal direction by a distance $\varepsilon$ and intersecting with the bottom circle of $\mathbf{C}$.

In the most straightforward case there are four distinct intersection points, dividing the circle into four segments which are alternately within and outside machining tolerance. Then the segment containing the $\mathrm{CC}$ point is used to determine the machined strip width. As $\beta$ varies, it is possible for the two segments machined within tolerance to merge, with the transition occurring where they just touch at a double intersection point. Consequently, the machined region can be classified broadly into three types: one single region, two connected regions or two disjoint regions.

The width function is determined numerically from the region or connected regions containing the CC point and its features, established by experimentation with thousands of quadrics and a range of machining tolerances, are as follows:

- Width $w$ may increase or decrease significantly when two disjoint regions merge into one or one region splits into two. So it is discontinuous in this case. If there is only a single region, then $w$ is continuous.

- In the discontinuous case, there are two local maxima within the range of $\beta$, corresponding to one region. Generally, they are at the two discontinuities.

- In the continuous case, there is one local maximum.

Figure 4 shows examples of discontinuous and continuous width functions, taken respectively from the inverse mouse and mouse surface machinings which were used previously to illustrate the quadric method (QM) for cutter orientation (Fan and Ball 2008). 

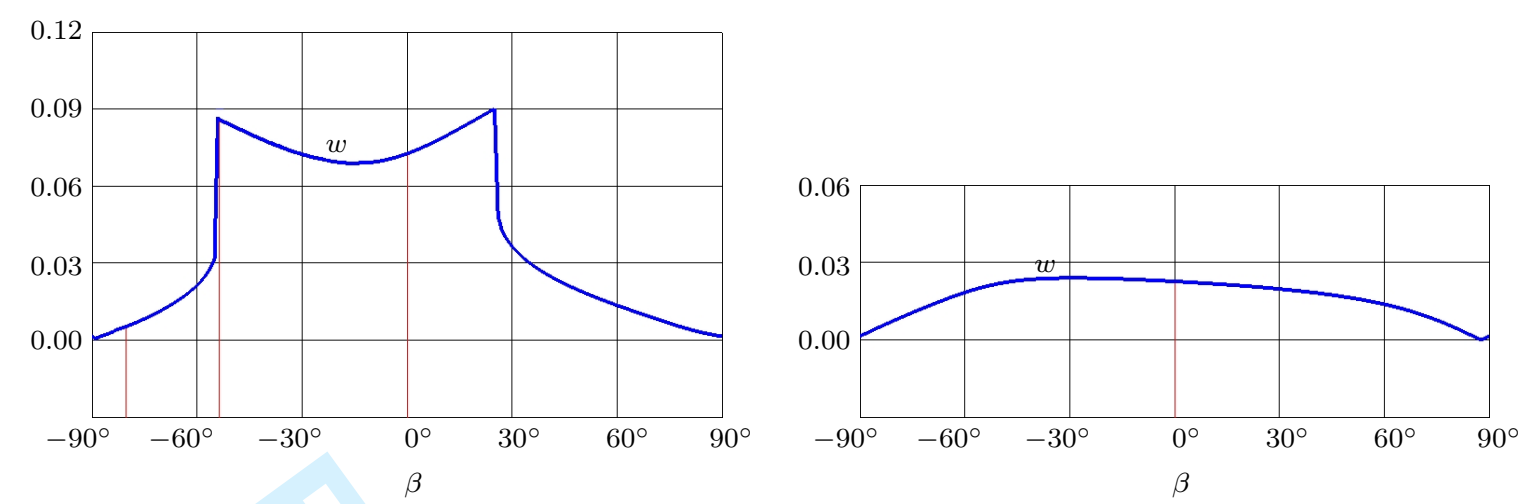

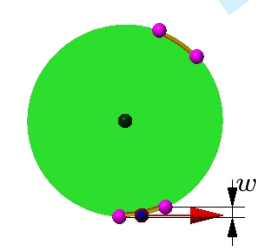

Two disjoint $\operatorname{regions}\left(\beta=-80^{\circ}\right)$

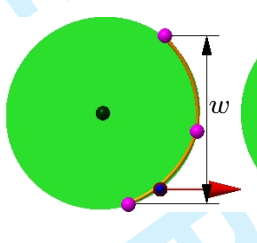

Two connected regions $\left(\beta=-52^{\circ}\right)$

(a)

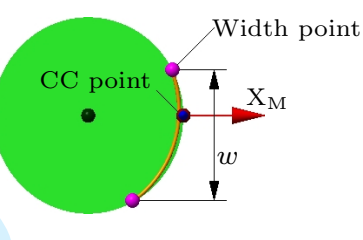

One single region $\left(\beta=0^{\circ}\right)$

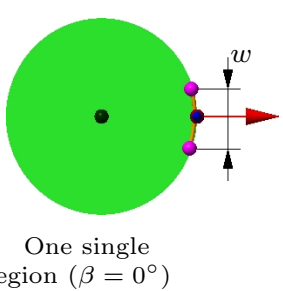

(b)

Figure 4. Width $w$ as a function of screw angle $\beta$ in (a) inverse mouse and (b) mouse surface machinings at $(0.5,0.75)$

Suppose that the cutter moves along a tool pass containing $m+1 \mathrm{CC}$ points. At the $j$ th CC point where $j=0,1,2, \ldots, m$, if the width is discontinuous, then the two local maxima are at $\beta_{h 1}(j)$ and $\beta_{h 0}(j)$, where $\beta_{h 1}(j)>\beta_{h 0}(j)$. If it is continuous, then the local maximum is at $\beta_{h}(j)$. In the next section, the angle $\beta$ will be optimised along the tool pass with consideration to the balance between the width and the smoothness of cutter movement.

\section{Smoothing screw angle}

The smoothness of the cutter movement is critical in 5-axis machining, and it is generally characterised by the rate of change of particular variables, such as the speed of the CC point (Lo 1999), the direction vector of the cutter axis (Li and Jerard 1994, Jun et al. 2003), and the angles of machine rotary axes (Affouard et al. 2004, Munlin et al. 2004). The speed of the CC point is determined mainly by the setting of the feed rate. The vector of the cutter axis and angles of the rotary axes are dependent on the lead angle $\alpha$ and the screw angle $\beta$. Since the width $w$ is a monotonic decreasing function of $\alpha$ (Jun et al. 2003, Fan 2006) and a much more complex function of $\beta$ as described in Section 2.2, this paper concentrates on maximising $w$ with a smooth $\beta$ along a tool pass. The method developed in the section is general, and could be applied to the smoothing of the angle $\alpha$ or other optimisation criteria.

The basic idea for smoothing the angle $\beta$ is to fit the local maxima with a cubic B-spline, which is the lowest possible degree for second derivative continuity (Piegl and Tiller 1997). To ensure there are no abrupt changes in the cutter movement, the first and second derivatives are kept within specified bounds. It should be noted that special care is needed for calculating the inverse kinematics of 5 -axis milling machines, due to singularities in the coordinate vector transformation. Consequently, a small deviation is 


\section{eptember 3, 2010

given to any tool orientation close to the singular positions to avoid abrupt changes in the machine rotary axes (Affouard et al. 2004, Munlin et al. 2004, Sørby 2007).

There are three cases to consider, dependent on whether the width is a discontinuous, continuous or hybrid function of the angle $\beta$ as it varies along the tool pass.

\subsection{Discontinuous case}

Consider the case when the width $w$ is discontinuous in terms of the angle $\beta$ at each CC point along the tool pass. First, $\beta_{h 1}(j), j=0,1,2, \ldots, m$ are fitted by the optimal screw angle $\beta_{H 1}$ described in cubic B-spline form as

$$
\beta_{H 1}(t)=\sum_{k=0}^{K} N_{k, 3}(t) H_{k}, \quad 0 \leq t \leq 1
$$

where $K+1$ is the number of control values $\left\{H_{k}\right\}$ with $K>2$, and the $\left\{N_{k, 3}(t)\right\}$ are the cubic B-spline basis functions defined on the unclamped, uniform knot vector (Piegl and Tiller 1997)

$$
T=\{\frac{-3}{K-2}, \frac{-2}{K-2}, \frac{-1}{K-2}, 0, \underbrace{\frac{1}{K-2}, \frac{2}{K-2}, \ldots, \frac{K-4}{K-2}, \frac{K-3}{K-2}}_{K-3 \text { internal knots }}, 1, \frac{K-1}{K-2}, \frac{K}{K-2}, \frac{K+1}{K-2}\} .
$$

It should be emphasised that the screw angle is a scalar function defined by a sequence $\left\{H_{k}\right\}$ of one-dimensional scalars whereas, in most B-spline applications, spatial curves are defined by a sequence of three-dimensional vectors. The cubic B-spline basis functions are identical in both cases.

The B-spline parameter $t$ is defined to vary linearly with the accumulated chord length $s$. Suppose $s=s_{j}$ and $t=t_{j}$ at the $j$ th CC point. Then $t_{j}=\frac{s_{j}}{s_{m}}, j=0,1,2 \ldots, m$. In addition, since $\frac{d s}{d t}$ is constant and $t_{m}=1, \frac{d s}{d t}=s_{m}, 0 \leq t \leq 1$.

Then, in fitting the screw angle along the tool pass, the objective function is set as

$$
\text { minimise } \quad z=\sum_{j=0}^{m}\left(\beta_{h 1}(j)-\sum_{k=0}^{K} N_{k, 3}\left(t_{j}\right) H_{k}\right) \text {. }
$$

To keep $\beta_{H 1}(t)$ on the side of $\beta_{h 1}(j)$ that corresponds to the one single region, there are constraints

$$
\beta_{h 1}(j)-\sum_{k=0}^{K} N_{k, 3}\left(t_{j}\right) H_{k} \geq 0, \quad j=0,1,2, \ldots, m
$$

To ensure smoothness of the fitting, it is necessary to control the rates of change of the screw angle along the tool pass. The first and second derivatives of $\beta_{H 1}$ with respect to 
$s, v_{H 1}(t)$ and $a_{H 1}(t)$, are related to the parametric derivatives of the B-spline as follows:

$$
\begin{aligned}
& v_{H 1}(t)=\frac{d \beta_{H 1}(t)}{d s}=\frac{d \beta_{H 1}(t)}{d t} \frac{d t}{d s}=\frac{1}{s_{m}} \sum_{k=0}^{K} N_{k, 3}^{\prime}(t) H_{k}, \\
& a_{H 1}(t)=\frac{d^{2} \beta_{H 1}(t)}{d s^{2}}=\frac{d^{2} \beta_{H 1}(t)}{d t^{2}}\left(\frac{d t}{d s}\right)^{2}=\frac{1}{s_{m}^{2}} \sum_{k=0}^{K} N_{k, 3}^{\prime \prime}(t) H_{k},
\end{aligned}
$$

where $N_{k, 3}^{\prime}(t)$ and $N_{k, 3}^{\prime \prime}(t)$ are respectively the first and second derivatives of $N_{k, 3}(t)$ with respect to $t$.

The principal method of control is to keep $v_{H 1}(t)$ within a specified range $[-V, V]$. It follows from (9) that

$$
s_{m}\left|v_{H 1}\left(t_{j}\right)\right|=\left|\sum_{k=0}^{K} N_{k, 3}^{\prime}\left(t_{j}\right) H_{k}\right| \leq s_{m} V, \quad j=0,1,2, \ldots, m .
$$

The above constraints not only control $v_{H 1}(t)$ but also help to keep $a_{H 1}(t)$ in check. However, further constraints are needed to counter the possibility of relatively high values of $\left|a_{H 1}(t)\right|$ at the ends of the spline. One control option is to specify zero first derivatives at the ends with $v_{H 1}(0)=v_{H 1}(1)=0$. Another option is to implement natural splines with $a_{H 1}(0)=a_{H 1}(1)=0$. A less restrictive option, adopted here, is to bound the values of $a_{H 1}(0)$ and $a_{H 1}(1)$ :

$$
s_{m}^{2}\left|a_{H 1}\left(t_{j}\right)\right|=\left|\sum_{k=0}^{K} N_{k, 3}^{\prime \prime}\left(t_{j}\right) H_{k}\right| \leq 4(K-2) s_{m} V, \quad j=0, m .
$$

It is proved in the Appendix A (with $D=s_{m} V, \frac{d \beta(t)}{d t}=s_{m} v_{H 1}(t)$ and $\frac{d^{2} \beta(t)}{d t^{2}}=$ $\left.s_{m}^{2} a_{H 1}(t)\right)$ that for each of the control options

$$
s_{m}^{2} \max \left|a_{H 1}(t)\right| \leq 4(K-2) s_{m} V .
$$

Consequently, to keep $a_{H 1}(t)$ within a specified range $[-A, A], K$ is assigned as the largest integer satisfying

$$
K \leq \frac{s_{m} A}{4 V}+2 .
$$

Then, it follows from (7), (8), (11) and (12) that the problem of optimising the screw 


\section{eptember 3,2010
Paqe $\mathbf{1 1}$ of $\mathbf{2 1}$ \\ International Journal of Production Research}

International Journal of Production Research

angle can be expressed as a constrained linear minimisation of the form:

$$
\begin{aligned}
\text { minimise } & z=\sum_{j=0}^{m}\left(\beta_{h 1}(j)-\sum_{k=0}^{K} N_{k, 3}\left(t_{j}\right) H_{k}\right), \\
\text { subject to } & \sum_{k=0}^{K} N_{k, 3}\left(t_{j}\right) H_{k} \leq \beta_{h 1}(j), \quad j=0,1,2, \ldots, m, \\
& \sum_{k=0}^{K} N_{k, 3}^{\prime}\left(t_{j}\right) H_{k} \leq s_{m} V, \quad j=0,1,2, \ldots, m, \\
- & \sum_{k=0}^{K} N_{k, 3}^{\prime}\left(t_{j}\right) H_{k} \leq s_{m} V, \quad j=0,1,2, \ldots, m, \\
& \sum_{k=0}^{K} N_{k, 3}^{\prime \prime}\left(t_{j}\right) H_{k} \leq 4(K-2) s_{m} V, \quad j=0, m, \\
- & \sum_{k=0}^{K} N_{k, 3}^{\prime \prime}\left(t_{j}\right) H_{k} \leq 4(K-2) s_{m} V, \quad j=0, m .
\end{aligned}
$$

The problem (15) can be further reformulated into the standard form of linear programming (Dantzig 1963), and then solved by the simplex method, which is efficient in solving linear problems with hundreds (even thousands) of variables and constraints (Vanderbei 2001). Hence, $\beta_{H 1}(t)$ is obtained. Similarly, replacing $\beta_{h 1}(j)$ by $\beta_{h 0}(j)$ and $H_{k}$ by $-H_{k}$ in (15) gives $\beta_{H 0}(t)$, the optimal screw angle fitting to $\beta_{h 0}(j)$, $j=0,1,2, \ldots, m$.

For example, consider the optimisation of the screw angles along the tool pass $v=0.75$ in the inverse mouse surface machining. Suppose $v_{H 1}(t)=\frac{d \beta_{H 1}(t)}{d s}$ and $v_{H 0}(t)=\frac{d \beta_{H 0}(t)}{d s}$ are to be kept within the range $\left[-1^{\circ} / \mathrm{mm}, 1^{\circ} / \mathrm{mm}\right]$ and $a_{H 1}(t)=\frac{d \beta_{H 1}^{2}(t)}{d s^{2}}$ and $a_{H 0}(t)=$ $\frac{d \beta_{H 0}^{2}(t)}{d s^{2}}$ within the range $\left[-0.3^{\circ} / \mathrm{mm}^{2}, 0.3^{\circ} / \mathrm{mm}^{2}\right]$. Given $s_{m}$ is approximately $125.4 \mathrm{~mm}$, then it follows from (14) that $K=11$ should be adopted for the fitting. Figure 5 shows the optimal screw angles $\beta_{H 1}(t)$ and $\beta_{H 0}(t)$, and confirms that the respective first and second derivatives are within the specified ranges. Whichever is used for the machining, there is no risk of abrupt changes in the cutter movement.

To construct the machining data at each $\mathrm{CC}$ point along the tool pass, the screw angle $\beta$ is evaluated from the B-spline approximation and the corresponding lead angle $\alpha$ is determined by the integrated orientation strategy of the quadric method (QM) (Fan and Ball 2008). As outlined in Section 2.2, the QM optimises $\alpha$ by placing the cutter $\mathbf{C}$ as close as possible to the upper quadric approximation of the design surface $\mathbf{S}$. Inevitably, the data smoothing reduces the width of the machined strip, but the QM minimises that reduction whilst guaranteeing there is no local interference. 


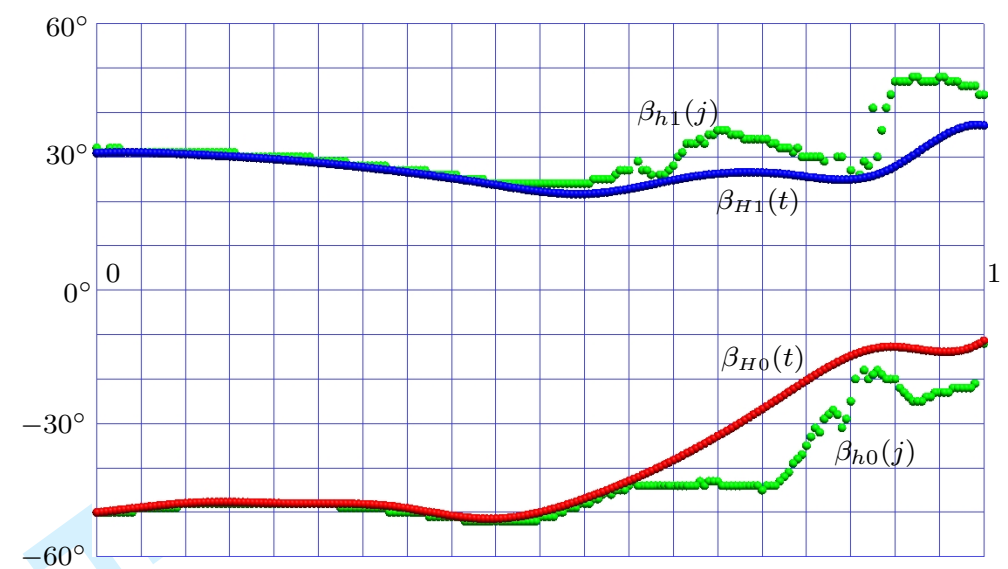

(a)

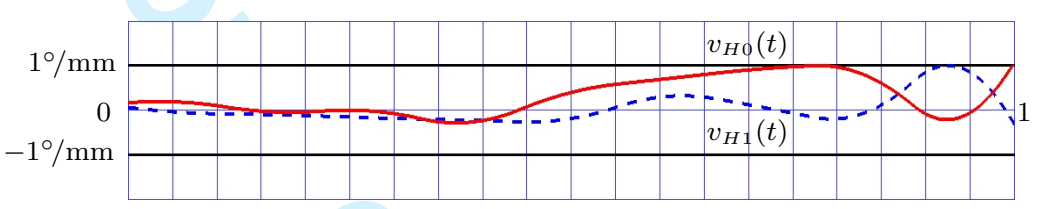

(b)

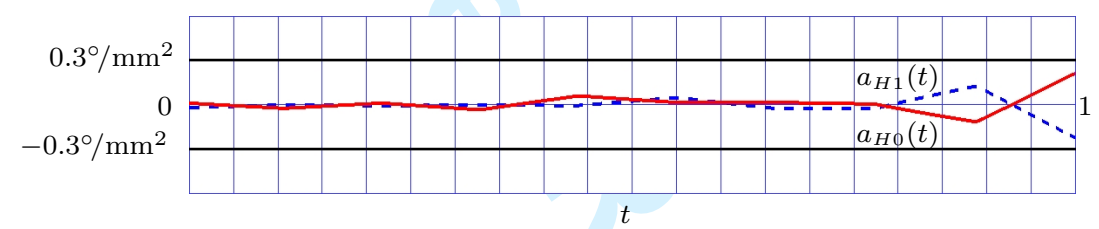

(c)

Figure 5. Optimisation along tool pass with $K=11$ showing (a) optimal screw angles (b) first and (c) second derivatives

\subsection{Continuous case}

Next, consider the case when the width is continuous in terms of the screw angle at each $\mathrm{CC}$ point along the tool pass. Two angle functions $\beta_{H 0}(t)$ and $\beta_{H 1}(t)$ are constructed in cubic B-spline form, which best fit $\beta_{h}(j), j=0,1,2, \ldots, m$, with limited first and second derivatives along the tool pass such that $\beta_{H 0}\left(t_{j}\right) \leq \beta_{h}(j) \leq \beta_{H 1}\left(t_{j}\right), j=0,1,2, \ldots, m$. More generally, any cubic spline of the form $\beta_{H \lambda}(t)=(1-\lambda) \beta_{H 0}(t)+\lambda \beta_{H 1}(t), 0 \leq \lambda \leq 1$, has first and second derivatives within the specified bounds. Unlike the discontinuous case, the sign of $\left(\beta_{H \lambda}(t)-\beta_{h}(t)\right)$ is not critical. Consequently, choosing $\lambda$ such that $\sum_{j=0}^{m}\left(\beta_{H \lambda}(t)-\beta_{h}(t)\right)=0$ or $\lambda=0.5$ would give reasonable, unbiassed fits but, for total compatibility with the discontinuous case, the choice is restricted to $\lambda=0$ or $\lambda=1$.

\subsection{Hybrid case}

Finally, consider the hybrid case when the width is discontinuous in terms of the screw angle at some $\mathrm{CC}$ points and continuous at others. If the width is discontinuous at the $j$ th CC point, then its local maxima are at $\beta_{h 1}(j)$ and $\beta_{h 0}(j)$, where $\beta_{h 1}(j)>\beta_{h 0}(j)$. If the width is continuous, then its local maxima are considered at $\beta_{h 1}(j)$ and $\beta_{h 0}(j)$ with 


\section{eptember 3,2010
Paqe $\mathbf{1 3}$ of $\mathbf{2 1}$ \\ International Journal of Production Research}

International Journal of Production Research

$\beta_{h 1}(j)=\beta_{h 0}(j)$. Consequently, two angle functions $\beta_{H 1}(t)$ and $\beta_{H 0}(t)$ are constructed in B-spline form, which best fit $\beta_{h 1}(j)$ and $\beta_{h 0}(j), j=0,1,2, \ldots, m$, as before.

The hybrid case essentially unifies the analysis and provides a general solution with two candidates, $\beta_{H 0}(t)$ and $\beta_{H 1}(t)$, for defining the screw angle. The choice for an individual machined pass could be determined by comparing $\min w_{H 0}(t)$ and $\min w_{H 1}(t)$ and adopting the one with the greater width. However, the overall surface quality is likely to be better by adopting a fixed preference for either $\beta_{H 0}(t)$ or $\beta_{H_{1}}(t)$ over the whole surface, since the spacing of the $\mathbf{P}_{i}$ 's will be more regular and the screw angle functions along successive $\mathbf{P}_{i}$ 's will be more compatible.

\section{Machining whole surface}

A method is proposed in this section to construct the machined strip $\mathbf{I}_{i}$ adjacent to a previously constructed $\mathbf{I}_{i-1}$, with an adaptation to construct the first strip $\mathbf{I}_{1}$. A discrete parametric definition is adopted for the profile of a machined strip and an iterative algorithm is proposed to maximise the step-over from $v_{i-1}$ to $v_{i}$, whilst ensuring there are no gaps between $\mathbf{I}_{i}$ and $\mathbf{I}_{i-1}$. The method is applicable to any construction of machined strips where the screw angle and lead angle functions are uniquely defined for all CC points along a tool pass, for all values of $v, 0 \leq v \leq 1$. Functions of particular interest in this paper are $\beta=0, \beta=\beta_{H 0}(t)$ or $\beta=\beta_{H 1}(t)$ with optimal $\alpha$; or $\beta=0$ with constant $\alpha$.

\subsection{Overlap between two machined strips}

The overlap between $\mathbf{I}_{i}$ and $\mathbf{I}_{i-1}$ is independent of the directions in which they are machined. So, to simplify the description and computational details, it is assumed that the tool passes have increasing $u$-values.

Suppose that at the $j$ th CC point along the tool pass $\mathbf{P}_{i}$, the parametric left and right width points are respectively at $\left(a_{i, j}, b_{i, j}\right)$ and $\left(c_{i, j}, d_{i, j}\right)$ as shown in figure 6 . Then the machined strip $\mathbf{I}_{i}$ is bounded by two sets of points $\left(a_{i, j}, b_{i, j}\right)$ and $\left(c_{i, j}, d_{i, j}\right)$, $j=0,1,2, \ldots, m(i)$, where $m(i)+1$ is the number of CC points along $\mathbf{P}_{i}$. The overlap distance $\Delta v_{i, j}$ between $\mathbf{I}_{i}$ and $\mathbf{I}_{i-1}$ at $\left(c_{i, j}, d_{i, j}\right)$ can be evaluated as

$$
\Delta v_{i, j}=\frac{c_{i, j}-a_{i-1, r}}{a_{i-1, r+1}-a_{i-1, r}}\left(b_{i-1, r+1}-b_{i-1, r}\right)+b_{i-1, r}-d_{i, j},
$$

where $r$ is determined by $a_{i-1, r} \leq c_{i, j}<a_{i-1, r+1}$. If $\Delta v_{i, j} \geq 0$, then $\mathbf{I}_{i}$ and $\mathbf{I}_{i-1}$ overlap at $\left(c_{i, j}, d_{i, j}\right)$ by a distance equal to $\Delta v_{i, j}$. Otherwise, there is a gap equal to $-\Delta v_{i, j}$.

Consequently, when constructing $\mathbf{I}_{i}$, a necessary condition is that $\Delta v_{i, j} \geq 0, j=$ $0,1,2, \ldots, m(i)$, i.e., there should be no gaps between $\mathbf{I}_{i}$ and $\mathbf{I}_{i-1}$.

\subsection{Determining step-over}

The primary objective in the construction of a machined strip $\mathbf{I}_{i}$ is to determine the parametric constant $v=v_{i}>v_{i-1}$ for the next tool pass $\mathbf{P}_{i}$. It is assumed that $b_{i-1}=\min \left\{b_{i-1, j}\right\}<1$, otherwise there would be no need to construct another tool pass. It is also assumed that the screw angle and lead angle functions are uniquely defined at all CC points along a tool pass, for all values of $v, 0 \leq v \leq 1$. 
If the machined strip along $v=1$ overlaps $\mathbf{I}_{i-1}$ without any gaps, then $v_{i}=1$ is adopted as an acceptable solution. Otherwise, the aim is to maximise the step-over from $v_{i-1}$ to $v_{i}$, within a tolerance $e_{p}$. Let $\delta\left(v_{i}\right)$ denote the minimum overlap of the machined strip along $v=v_{i}$ with $\mathbf{I}_{i-1}, 1 \geq v_{i} \geq v_{i-1}$. Then $b_{i-1}<1, \delta\left(b_{i-1}\right)>0$ and $\delta(1)<0$. Consequently, by successive bisection of the interval $\left[b_{i-1}, 1\right]$, it is possible to find an interval $\left[v_{i}, v_{i}^{*}\right]$ where $\delta\left(v_{i}\right) \geq 0, \delta\left(v_{i}^{*}\right)<0$ and $0<v_{i}^{*}-v_{i} \leq e_{p}$. Of course, there are generally more computationally efficient ways of finding $v_{i}$.

For example, an iterative algorithm is proposed to find $v_{i}$ which aims to minimise the overlap with $\mathbf{I}_{i-1}$ rather than maximise the step-over. Let $\min \left\{w_{i-1}\right\}$ denote the minimum width of $\mathbf{I}_{i-1}$, then the $v$-value of $\mathbf{P}_{i}$ is initialised at

$$
v_{i, 0}=v_{i-1}+\min \left\{w_{i-1}\right\}
$$

At the $q$ th iteration, suppose that the minimum overlap distance between the two strips is $\delta v_{i, q}=\min \left\{\Delta v_{i, j}\right\}$. If $0 \leq \delta v_{i, q} \leq e_{p}$, then the iteration is terminated. Otherwise, the $v$-value of $\mathbf{P}_{i}$ at the $(q+1)$ th iteration is determined by

$$
v_{i, q+1}=v_{i, q}+\delta v_{i, q}-e_{p} / 2
$$

For total computational robustness, it is advisable to incorporate within the software solution a binary search to find $v_{i}$, which can be triggered whenever there is slow convergence.

The above algorithm must be adapted for the first machined strip $\mathbf{I}_{1}$, where there is not a previously constructed strip. In this case a dummy machined strip is constructed along $v=0$. Let $\min \left\{w_{0}\right\}$ denote the minimum width of the whole strip and $b_{0}$ denote the minimum width of that part with $v \geq 0$. Then, in the iterative process to find $v_{i}$, equations (16) and (17) are adapted to

$$
\begin{aligned}
\Delta v_{1, j} & =-d_{1, j} \\
v_{1,0} & =-b_{0}+\min \left\{w_{0}\right\} .
\end{aligned}
$$

Consequently, the first machined strip will overlap $v=0$ such that the minimum overlap is within tolerance. 


\section{eptember 3,2010
Paqe $\mathbf{1 5}$ of $\mathbf{2 1}$ \\ International Journal of Production Research}

International Journal of Production Research

\section{Inverse mouse surface machining}

The proposed integrated method (IM) was implemented using Visual $\mathrm{C}++{ }^{\circledR}$ and OpenGL ${ }^{\circledR}$ on a personal computer with an Inter $^{\circledR}$ Core $^{\mathrm{TM}} 2$ Duo $2.33 \mathrm{GHz}$ Processor and $2.00 \mathrm{~GB}$ of RAM under Windows ${ }^{\circledR}$ XP Professional SP2 operating system. The implementation comprises four modules for lead angle optimisation, screw angle optimisation, width evaluation and step-over determination. The cutter is a flat-end cutter with radius $R=4 \mathrm{~mm}$, machining tolerance $\varepsilon=0.01 \mathrm{~mm}$, allowable overlap distance $e_{p}=2 \mathrm{e}^{-4}$, and accuracies of point evaluation $e_{1}=e_{2}=1 \mathrm{e}^{-5} \mathrm{~mm}$. It took 6.32 minutes to generate 16 tool passes to cover the entire inverse mouse surface as shown in figure $7(\mathrm{a})$.

The tool path has been checked by Toolpath Verification in PowerMILL ${ }^{\circledR}$ 9.0, a world leading CAM software package, and there are no gouges/collisions reported. The machining was also simulated using ViewMILL in PowerMILL and figure 7(b) illustrates the shiny rendered image. It should be noted that ViewMILL employs a dynamic flute model, rather than a cylindrical surface, to define the geometry of the cutter and produces photorealistic images which correspond almost perfectly to the results of physical machinings (Liu et al. 2005). Comparing figures 7(a) and 7(b), it can be seen that they have very similar strip patterns although the ViewMILL shows up some minor surface irregularities, well within the machining tolerance, just as one would get in the physical machining.

Table 1 gives the $v$-values of the corresponding tool passes, and figure 8(a) illustrates the machined strips in parametric space. Suppose that the surface is physically machined with a feed rate of $250 \mathrm{~mm} / \mathrm{min}$, then the total machining time is approximately 8.26 minutes.

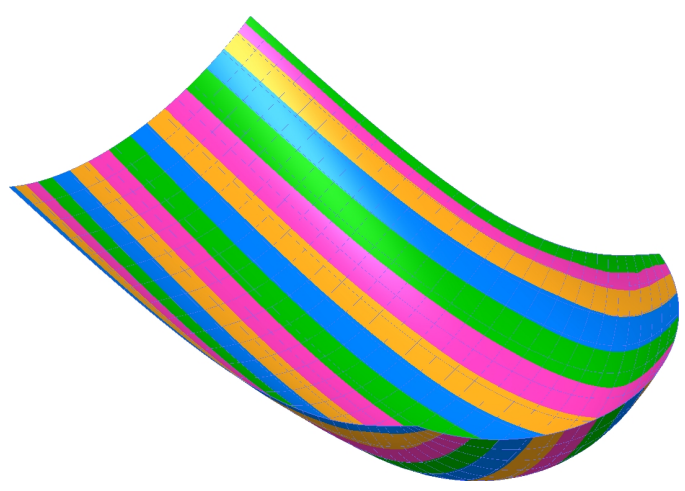

(a)

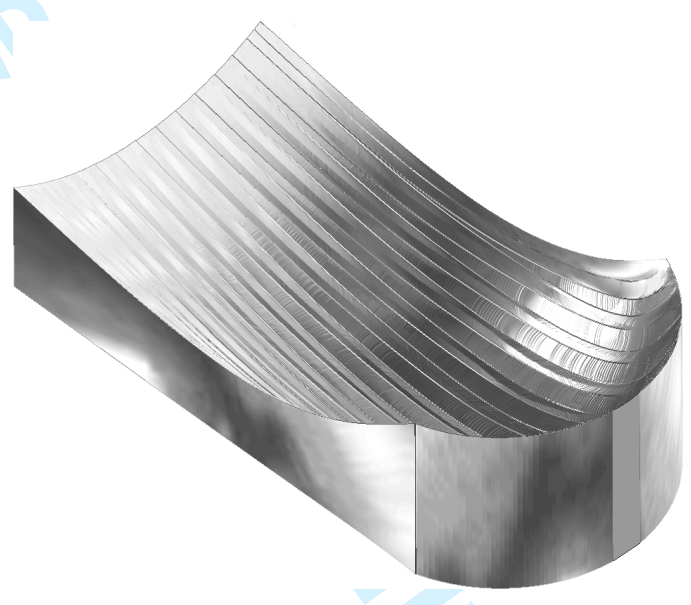

(b)

Figure 7. Inverse mouse surface machining showing (a) machined strips and (b) simulation result

Table 1. $v$-values of tool passes

\begin{tabular}{ccccccccc}
\hline No. & 1 & 2 & 3 & 4 & 5 & 6 & 7 & 8 \\
\hline$v$ & 0.0110 & 0.0411 & 0.0881 & 0.1417 & 0.2068 & 0.2741 & 0.3482 & 0.4293 \\
\hline No. & 9 & 10 & 11 & 12 & 13 & 14 & 15 & 16 \\
\hline$v$ & 0.5104 & 0.5872 & 0.6659 & 0.7425 & 0.8206 & 0.8935 & 0.9551 & 0.9862 \\
\hline
\end{tabular}


Table 2 compares of the IM with some alternative machining strategies introduced in Section 1.2 for machining the inverse mouse surface, and figure 8 illustrates the corresponding machined strips in parametric space. In some published 5-axis machining methods (Gray et al. 2003, 2005, Hosseinkhani et al. 2007), the screw angle $\beta$ is kept at $0^{\circ}$ and the lead angle $\alpha$ is optimised by placing the cutter as close as possible to the design surface. The computational time for this method to generate 24 tool passes to cover the

Table 2. Comparison of alternative machining methods in inverse mouse surface machining

\begin{tabular}{lccc}
\hline & Number of passes & Computational time(min) & Machining time(min) \\
\hline Integrated method & 16 & 6.32 & 8.26 \\
$\beta=0^{\circ}$ with optimal $\alpha$ & 24 & 2.41 & 12.40 \\
$\beta=0^{\circ}, \alpha=10^{\circ}$ & 50 & 3.55 & 25.80 \\
\hline
\end{tabular}

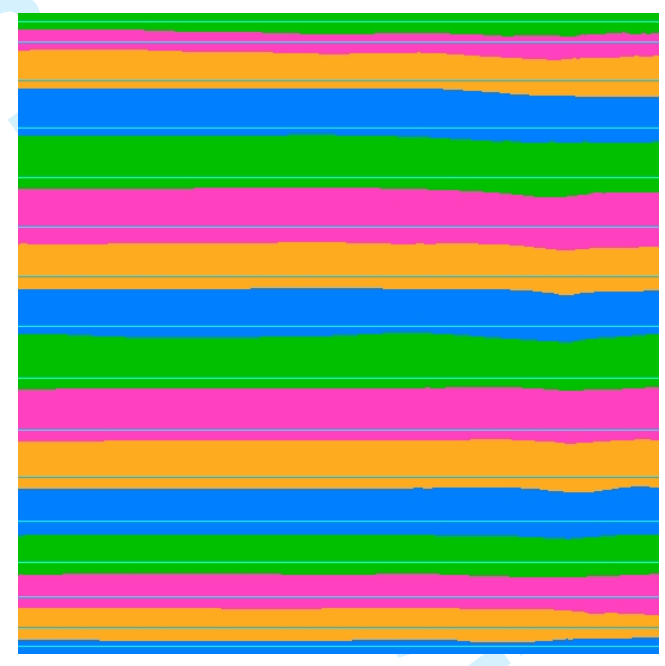

(a)

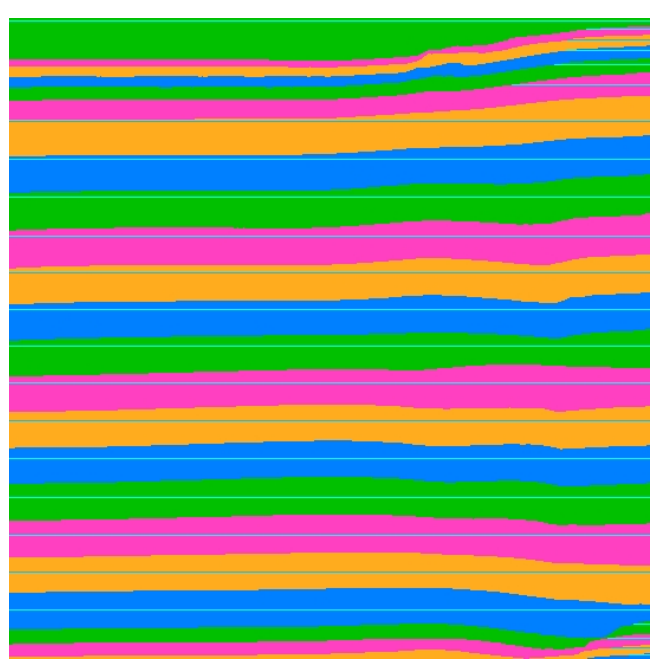

(b)

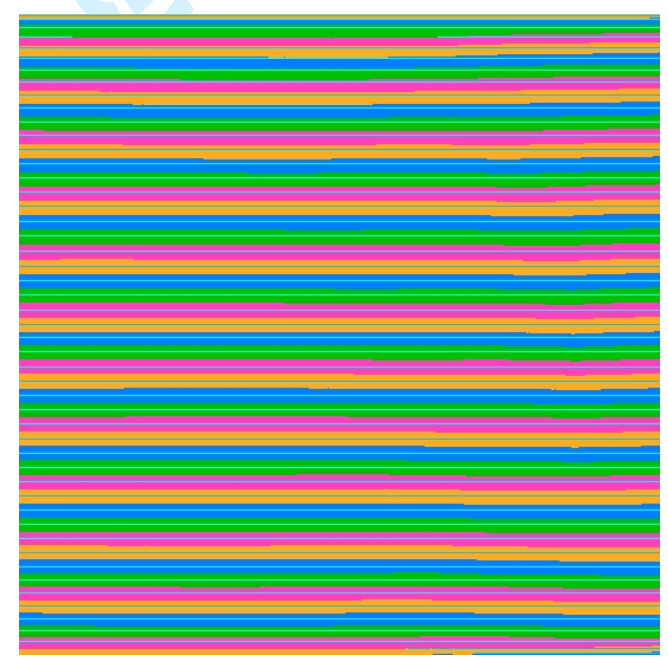

(c)

Figure 8. Comparison of machined strips in parametric space showing (a) Integrated method (16 passes), (b) $\beta=0^{\circ}$ with optimal $\alpha$ (24 passes), and (c) $\beta=0^{\circ}, \alpha=10^{\circ}$ (50 passes) 


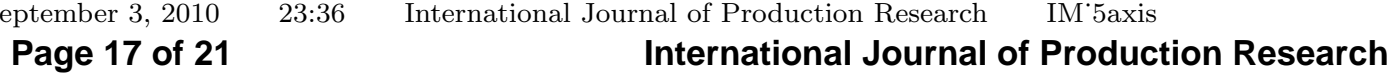

entire surface is 2.41 minutes, and the machining time is 12.40 minutes. Alternatively, if the surface is machined by the conventional 5 -axis method with $\alpha=10^{\circ}$ and $\beta=0^{\circ}$ (Choi and Jerard 1998), it will take 3.55 minutes to generate 50 tool passes and 25.80 minutes to physically machine the surface.

The example illustrates the potential efficiency savings of the IM compared to the other strategies, with reductions in the machining times of $33 \%$ and $68 \%$ respectively. The simple practical explanation for the improvement is that the two orientation angles of the cutter are fully exploited in the IM, so that the cutter surface is optimally close to the design surface, subject only to the constraint of having a smooth cutter movement. Further, comparing the machined strips in figure 8, it can be seen that those in 8 (a) are as regular as those in $8(\mathrm{c})$ and more regular than those in $8(\mathrm{~b})$, which suggest the machined surface quality is as good with the IM as with the other strategies.

The one disadvantage of the IM is the extra computational time, with the bulk of that time (about $0.2 \mathrm{~min} /$ pass) spent on searching for the optimal screw angle. Certainly, there is a scope for improving the search algorithm but the extra cost of computation is already small compared to the valuable savings in 5-axis machining time. Indeed, in all cases, the extra computational time is outweighed by the reduction in machining time without any consideration to their relative value.

\section{Conclusions}

In this paper, the integrated method (IM) has been developed for tool path generation in 5-axis machining. Distinctive from existing methods, both the machined strip width and the smoothness of cutter movement are considered in determining cutter orientation along a tool pass. Further, the next tool pass is positioned as far as permissible from the previous one. It follows that the two cutter orientation angles are fully exploited along the tool passes and the step-over distances are maximised under the constraint of smooth cutter movements. Consequently, the IM is more efficient and the numerical simulations in Section 5 have shown that there can be a significant reduction in the number of tool passes required by the IM compared to other methods.

In the paper, the IM has been combined with the quadric method (QM) in iso-parametric machining with a flat-end cutter. However, the IM can be extended easily to machining with a fillet-end cutter, according to the geometric equivalency between the flat-end and fillet-end cutters (Yoon 2003, Fan and Ball 2008). Also, it is applicable to other orientation strategies with different machining mechanisms, based on the width evaluation in physical space (Marciniak 1991). Finally, by formulating the possible constraints from collision avoidance into a linear form, an efficient, smooth and safe tool path can be obtained.

\section{Acknowledgements}

The first author would like to acknowledge the financial support of the Overseas Research Students (ORS) award from Universities UK and the Research Scholarship from the School of Mechanical Engineering at the University of Birmingham. The authors thank Martin Loftus and Robert Cripps at the University of Birmingham, Jonathan Corney at the University of Strathclyde and Steve Hobbs at Delcam PLC for their valuable comments and suggestions. 


\section{Appendix A.}

Suppose that $\beta(t), 0 \leq t \leq 1$ is a uniform cubic B-spline comprising $(K-2)$ segments, $K>2$, where $\max \left|\frac{d \bar{\beta}(t)}{d t}\right| \leq D$.

$$
\begin{aligned}
& \text { If } \quad \text { (i) } \frac{d \beta(0)}{d t}, \frac{d \beta(1)}{d t}=0, \\
& \text { (ii) } \frac{d^{2} \beta(0)}{d t^{2}}, \frac{d^{2} \beta(1)}{d t^{2}}=0, \\
& \text { or } \quad\left(\text { iii) }\left|\frac{d^{2} \beta(0)}{d t^{2}}\right|,\left|\frac{d^{2} \beta(1)}{d t^{2}}\right| \leq 4(K-2) D,\right. \\
& \text { then } \max \left|\frac{d^{2} \beta(t)}{d t^{2}}\right| \leq 4(K-2) D .
\end{aligned}
$$

Proof: $\frac{d^{2} \beta(t)}{d t^{2}}$ is a uniform linear B-spline comprising $(K-2)$ segments, varying linearly from $\frac{d^{2} \beta\left(\frac{l}{K-2}\right)}{d t^{2}}$ to $\frac{d^{2} \beta\left(\frac{l+1}{K-2}\right)}{d t^{2}}, \frac{l}{K-2} \leq t \leq \frac{l+1}{K-2}, l=0,1, \cdots, K-3$. It follows that the maximum value of $\left|\frac{d^{2} \beta(t)}{d t^{2}}\right|$ must occur at one of the segment ends.

First suppose that the maximum of $\left|\frac{d^{2} \beta(t)}{d t^{2}}\right|$ occurs at $t=0$. Then result follows immediately in cases (ii) and (iii). In case (i)

$$
\frac{d^{2} \beta(t)}{d t^{2}}=(1-(K-2) t) \frac{d^{2} \beta(0)}{d t^{2}}+(K-2) t \frac{d^{2} \beta\left(\frac{1}{K-2}\right)}{d t^{2}}, \quad 0 \leq t \leq \frac{1}{K-2},
$$

where

$$
-\left|\frac{d^{2} \beta(0)}{d t^{2}}\right| \leq \frac{d^{2} \beta\left(\frac{1}{K-2}\right)}{d t^{2}} \leq\left|\frac{d^{2} \beta(0)}{d t^{2}}\right|
$$

Integrating with $\frac{d \beta(0)}{d t}=0$ gives

$$
\frac{d \beta(t)}{d t}=\left(t-\frac{1}{2}(K-2) t^{2}\right) \frac{d^{2} \beta(0)}{d t^{2}}+\frac{1}{2}(K-2) t^{2} \frac{d^{2} \beta\left(\frac{1}{K-2}\right)}{d t^{2}}, \quad 0 \leq t \leq \frac{1}{K-2} .
$$

Minimum range of $\frac{d \beta(t)}{d t}$ over $\left[0, \frac{1}{K-2}\right]$ occurs when $\frac{d^{2} \beta\left(\frac{1}{K-2}\right)}{d t^{2}}=-\frac{d^{2} \beta(0)}{d t^{2}}$. Then

$$
\frac{d \beta(t)}{d t}=\left(t-(K-2) t^{2}\right) \frac{d^{2} \beta(0)}{d t^{2}}, \quad 0 \leq t \leq \frac{1}{K-2}
$$

and $\frac{d \beta(t)}{d t}$ ranges from 0 to $\frac{1}{4(K-2)} \frac{d^{2} \beta(t)}{d t^{2}}$.

Since $\max \left|\frac{d \beta(t)}{d t}\right| \leq D$, if follows that

$$
\max \left|\frac{d^{2} \beta(t)}{d t^{2}}\right|=\max \left|\frac{d^{2} \beta(0)}{d t^{2}}\right| \leq 4(K-2) D
$$




\section{eptember 3,2010
Page $\mathbf{1 9}$ of $\mathbf{2 1}$

The proof is similar at $t=1$.

Now suppose that the maximum of $\left|\frac{d^{2} \beta(t)}{d t^{2}}\right|$ occurs at $t=\frac{l}{K-2}, 1 \leq l \leq K-3$. Then

$$
-\left|\frac{d^{2} \beta\left(\frac{l}{K-2}\right)}{d t^{2}}\right| \leq \frac{d^{2} \beta\left(\frac{l-1}{K-2}\right)}{d t^{2}}, \quad \frac{d^{2} \beta\left(\frac{l+1}{K-2}\right)}{d t^{2}} \leq\left|\frac{d^{2} \beta\left(\frac{l}{K-2}\right)}{d t^{2}}\right| .
$$

The minimum range of $\frac{d \beta(t)}{d t}$ over $\left[\frac{l-1}{K-2}, \frac{l+1}{K-2}\right]$ occurs when

$$
\frac{d^{2} \beta\left(\frac{l-1}{K-2}\right)}{d t^{2}}=\frac{d^{2} \beta\left(\frac{l+1}{K-2}\right)}{d t^{2}}=-\frac{d^{2} \beta\left(\frac{l}{K-2}\right)}{d t^{2}} .
$$

Then $\frac{d \beta(t)}{d t}$ ranges from

$$
\frac{d \beta\left(\frac{l}{K-2}\right)}{d t}-\frac{1}{4(K-2)} \frac{d^{2} \beta\left(\frac{l}{K-2}\right)}{d t^{2}} \text { to } \frac{d \beta\left(\frac{l}{K-2}\right)}{d t}+\frac{1}{4(K-2)} \frac{d^{2} \beta\left(\frac{l}{K-2}\right)}{d t^{2}} .
$$

Since $\frac{d \beta(t)}{d t}$ ranges at most from $-D$ to $D$, it follow that

$$
\max \left|\frac{d^{2} \beta(t)}{d t^{2}}\right|=\max \left|\frac{d^{2} \beta\left(\frac{l}{K-2}\right)}{d t^{2}}\right| \leq 4(K-2) D
$$

\section{References}

Affouard, A., et al., 2004. Avoiding 5-axis singularities using tool path deformation. International Journal of Machine Tools and Manufacture, 44 (4), 415-425.

Bedi, S., Gravelle, S., and Chen, Y.H., 1997. Principal curvature alignment technique for machining complex surfaces. ASME Journal of Manufacturing Science and Engineering, 119, 756-765.

Chiou, C.J. and Lee, Y.S., 2002. A machining potential field approach to tool path generation for multi-axis sculptured surface machining. Computer-Aided Design, 34 (5), 357-371.

Choi, B.K. and Jerard, R.B., 1998. Sculptured Surface Machining - Theory and Applications. Dordrecht, Netherlands: Kluwer Academic Publishers.

Dantzig, G.B., 1963. Linear Programming and Extensions. Princeton, USA: Princeton University Press.

Fan, J., 2006. Geometric modelling of 5-axis sculptured surface machining. Thesis (PhD). The University of Birmingham.

Fan, J. and Ball, A., 2008. Quadric method for cutter orientation in five-axis sculptured surface machining. International Journal of Machine Tools and Manufacture, 48 (7-8), 788-801.

Farin, G., 2002. Curves and surfaces for CAGD - A Practical Guide. 5th San Francisco, USA: Morgan kaufmann. 
Gray, P.J., Bedi, S., and Ismail, F., 2003. Rolling ball method for 5-axis surface machining. Computer-Aided Design, 35 (4), 347-357.

Gray, P.J., Bedi, S., and Ismail, F., 2005. Arc-intersect method for 5-axis tool positioning. Computer-Aided Design, 37 (7), 663-674.

Hosseinkhani, Y., Akbari, J., and Vafaeesefat, A., 2007. Penetration-elimination method for five-axis CNC machining of sculptured surfaces. International Journal of Machine Tools and Manufacture, 47 (10), 1625-1635.

Jensen, C.G., Red, W.E., and Pi, J., 2002. Tool selection for five-axis curvature matched machining. Computer-Aided Design, 34 (3), 251-266.

Jun, C.S., Cha, K., and Lee, Y.S., 2003. Optimizing tool orientations for 5-axis machining by configuration-space search method. International Journal of Machine Tools and Manufacture, 35 (6), 549-566.

Li, H. and Feng, H.Y., 2004. Efficient five-axis machining of free-form surfaces with constant scallop height tool paths. International Journal of Production Research, 42 (12), 2403-2417.

Li, S.X. and Jerard, R.B., 1994. 5-axis machining of sculptured surface with a flat-end cutter. Computer-Aided Design, 26 (3), 165-178.

Liu, N., Loftus, M., and Whitten, A., 2005. Surface finish visualisation in high speed, ball nose milling applications. International Journal of Machine Tools and Manufacture, 45 (10), 1152-1161.

Lo, C.C., 1999. Real-time generation and control of cutter path for 5-axis CNC machining. International Journal of Machine Tools and Manufacture, 30 (3), 471-488.

Marciniak, K., 1991. Geometric Modelling for Numerically Controlled Machining. Oxford, UK: Oxford University Press.

Munlin, M., Makhanov, S.S., and Bohez, E.L.J., 2004. Optimization of rotations of a five-axis milling machine near stationary points. Computer-Aided Design, 36 (12), $1117-1128$.

Piegl, L.A. and Tiller, W., 1997. The NURBS Book. 2nd Berlin, Germany: Springer.

Rao, A. and Sarma, R., 2000. On local gouging in five-axis sculptured surface machining using flat-end tools. Computer-Aided Design, 32 (7), 409-420.

Rao, N., Ismail, F., and Bedi, S., 2000. Integrated tool positioning and tool path planning for five-axis machining of sculptured surface. International Journal of Production Research, 38 (12), 2709-2724.

Sørby, K., 2007. Inverse kinematics of five-axis machines near singular configurations. International Journal of Machine Tools and Manufacture, 47 (2), 299-603.

Tournier, C. and Duc, E., 2005. Iso-scallop tool path generation in 5-axis milling. International Journal of Advanced Manufacturing Technology, 25 (9-10), 867-875.

Vanderbei, R.J., 2001. Linear Programming: Foundations and Extensions. 2nd Berlin, Germany: Springer.

Vickers, G.W. and Quan, K.W., 1989. Ball-mills versus end-mills for curved surface milling. ASME Journal of Engineering for Industry, 111 (4), 24-22.

Wang, N. and Tang, K., 2007. Automatic generation of gouge-free and angularvelocity-compliant five-axis toolpath. Computer-Aided Design, 39 (10), 841-852.

Warkentin, A., Ismail, F., and Bedi, S., 2000. Multi-point tool positioning strategy for 5-axis machining of sculptured surface. Computer Aided Geometric Design, 17 (1), 83-100.

Yoon, J.H., 2003. Tool tip gouging avoidance and optimal tool positioning for 5-axis sculptured surface machining. International Journal of Production Research, 41 (10), $2121-2142$. 
Yoon, J.H., Pottmann, H., and Lee, Y.S., 2002. Locally optimal cutting positions for 5-axis sculptured surface machining. Computer-Aided Design, 35 (1), 69-81. 